\title{
MS02-03 | FragmaX - The New Fragment Screening Facility of MAX IV LABORATORY
}

Mueller, Uwe (MAX IV Laboratory, Lund, SWE); Lima, Gustavo (MAX IV Laboratory, Lund, SWE); Knecht, Wolfgang (Lund University, Lund, SWE); Logan, Derek (Saromics Biostructures AB, Lund, SWE); Sjögren, Tove (AstraZeneca, Mölndal, SWE)

Crystallographic fragment screening is a powerful tool to probe the functional surface of proteins and to assist pharmaceutical industry within their drug discovery process to identify small molecular binders. FragMAX is currently developed to a high throughput x-ray diffraction fragment screening facility using BioMAX, the first MX beamline at MAX IV Laboratory. FragMAX entails the infrastructure to produce apo-target protein crystals, to incubate those crystals with fragments via soaking or co-crystallisation, harvest the crystals and submit them to an screening experiment at BioMAX. The derived experimental data are automatically processed and further analysed in order to provide a initial ranking for potential hits to the user automatically. All experimental and analysis data are stored within a dedicated data base and are presented within an in house developed user interface. FragMAX is developed and operated in close colaboration with the Lund protein production platform LP3, AstraZeneca and Saromics Biostructures as a user facility for academic and industrial users. 